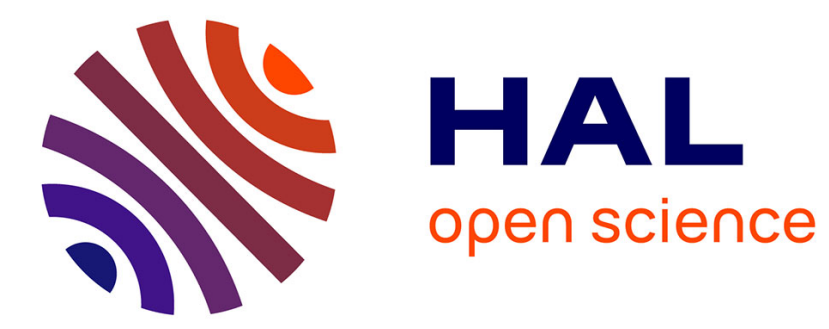

\title{
Encore des Mosaïques de Bellérophon
}

Jocelyn M.C. Toynbee

\section{To cite this version:}

Jocelyn M.C. Toynbee. Encore des Mosaïques de Bellérophon. Gallia - Fouilles et monuments archéologiques en France métropolitaine, 1958, 16 (2), pp.262-266. 10.3406/galia.1958.2233 . hal01924559

\section{HAL Id: hal-01924559 \\ https://hal.science/hal-01924559}

Submitted on 6 Feb 2020

HAL is a multi-disciplinary open access archive for the deposit and dissemination of scientific research documents, whether they are published or not. The documents may come from teaching and research institutions in France or abroad, or from public or private research centers.
L'archive ouverte pluridisciplinaire HAL, est destinée au dépôt et à la diffusion de documents scientifiques de niveau recherche, publiés ou non, émanant des établissements d'enseignement et de recherche français ou étrangers, des laboratoires publics ou privés.

\section{(이) $\$$}

Distributed under a Creative Commons Attribution - NonCommercial - NoDerivatives $\mid 4.0$ 


\section{Encore des Mosaiques de Bellérophon}

M. Pierre Amandry a publié dans la Revue Archéologique de 1956 (6e série, XLVIII, p. 155-156) une liste de dix mosaïques représentant Bellérophon, en ajoutant deux, celles de Parndorf (Aulriche) et de Constantinople, aux huit que j'avais citées dans Gallia en
1955 (XIII, p. 91-97). Depuis, deux autres mosaïques sont venues à ma connaissance qui représentent le héros l'une certainement, l'autre très probablement.

La première mosaïque, pavement polychrome trouvé dans une villa près de

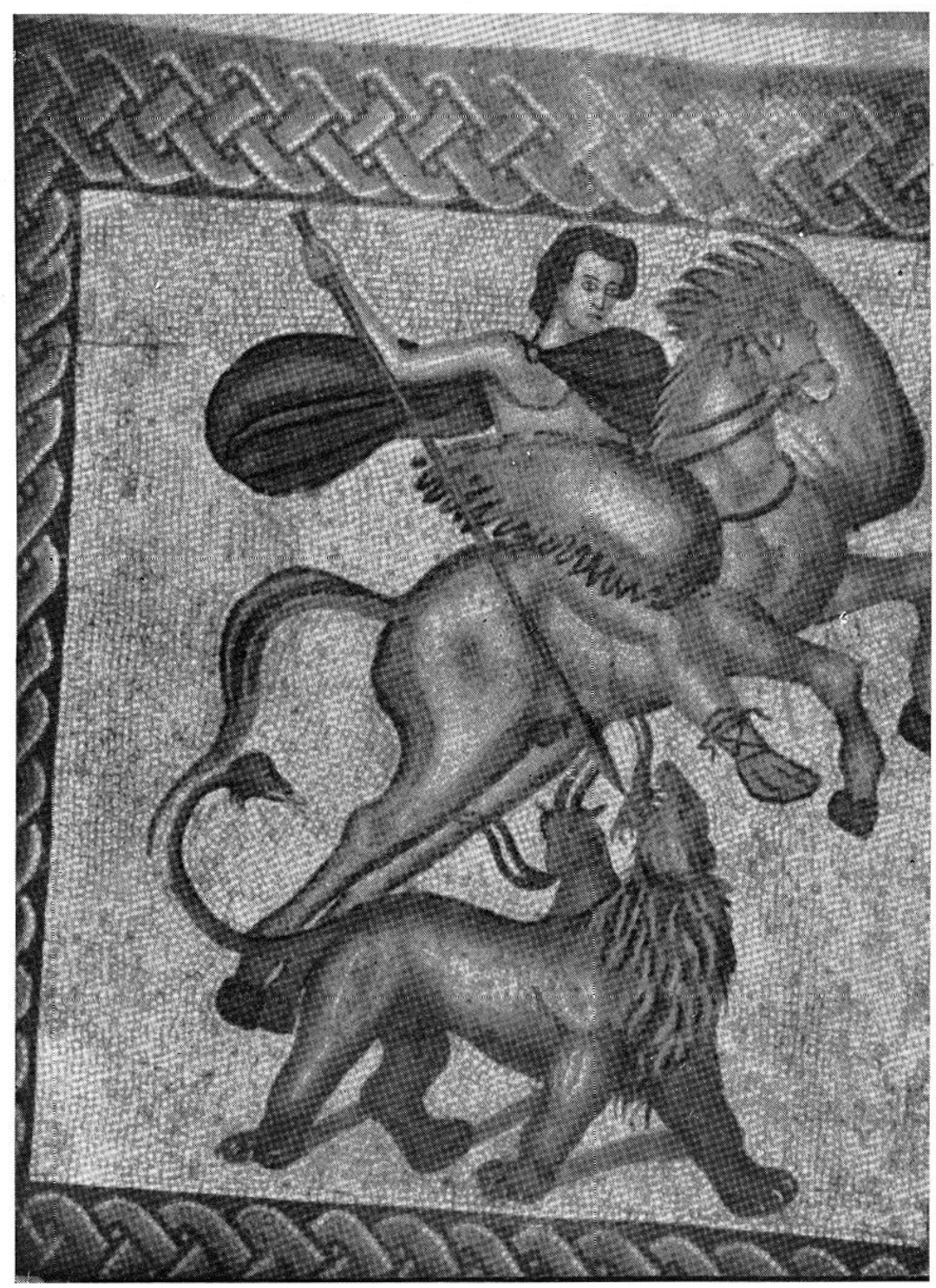

Fig. 1. - Panneau central de la mosaïque de Gerona (Musée de Barcelone). 
Gerona (Espagne), actuellement au Musée de Barcelone, a été récemment publicé par J.-C. Baroja ${ }^{1}$. La scène (fig. 1) se déploie dans un champ rectangulaire à fond blanc, encadré d'une bande jaune décorée d'une tresse à quatre cordons. Bellérophon monté sur Pégase vole vers la droite et pointe sa lance, tenue en diagonale de la main droite, sur la gueule de lion de la Chimère. Celle-ci se dirige à grands pas vers la droite, sa tête de lion étant relevée vers l'arrière pour faire face à l'assaillant. La tête de chèvre qui lui sort des épaules, juste au-dessus de la patte antérieure droite, regarde vers la gauche. La tête de lion et la tête de chèvre vomissent toutes deux une flamme à double pointe de couleur rouge foncé. La queue du monstre se termine en une tête de scrpent bombée et barbue. Bellérophon n'est vêtu que d'un manteau attaché par une fibule ronde à l'épaule droite et dont la couleur rivalise avec le rouge foncé de ses cheveux, et de sandales aux lanières noires. Sa chair est d'un crème soutenu, son expression grave et concentrée. Pégase porte robe grise, crinière grise, ailes grises ; les rênes sont marron. Son aspect est farouche : il jette un regard de côté vers la proie que vise son maître. La Chimère a têtes, corps et queue tout entière d'un fauve profond, avec des raies noires pointant entre les mèches de sa crinière de lion. Les ombres projetées par les pattes du monstre sont grises.

L'effet général de la gamme des couleurs employée est sobre mais décoratif. Les figures de l'homme et des animaux sont vigoureusement dessinées, bien modelées et les ombres sont traitées de façon naturaliste. C'est une œuvre

(1) España primitiva y romana, 1957, pl. 7, face à p. 288 . à trois dimensions, où l'espace et l'atmosphère sont représentés et qui fait un contraste frappant avec le groupe du Bellérophon de Lullingstone, œuvre du rve siècle, strictement conçue à deux dimensions ${ }^{2}$. Néanmoins, le pavement de Barcelone trahit quelques erreurs de disposition. Comme à Lullingstone, l'artiste a donné aux héros une seule jambe droite, bien que le pied gauche ait pu et dû apparaître derrière les antérieurs du cheval ; la patte antérieure gauche de la Chimc̀re ne projette pas d'ombre. Le mélange d'exactitude et de naturalisme dans le dessin et le modelé avec une composition défectueuse convient à la datation du irre siècle que Baroja assigne à cette mosaïque.

La seconde mosaïque qui doit être, je crois, ajoutée à notre liste est le médaillon central d'un grand pavement rectangulaire polychrome trouvé en 1728 dans une villa gallo-romaine à IIerzogenbuchsee, à l'Est de Soleure (Suisse) ${ }^{3}$. Une panthère, sautant dans l'un des demi-mćdaillons secondaires, a été reconstituće à une époque récente et est conservée au Musée historique bernois, où se trouve également un dessin coloré de Stürler (no 18.525) représentant la mosaïque entière lors de sa découverte, y compris la scène centrale aujourd'hui disparue (fig. 2) ${ }^{4}$. Celle-ci montre Pégase volant vers la droite, portant un jeune cavalier. Ge dernier, bien qu'assis à califourchon, se retourne vers le spectateur; il porte un manteau attaché sur l'épaule droite et dont une extrćmitć s'envole à droite de sa tête, tandis

(2) Gallia, XIII (1955), p. 95, fig. 4.

(3) A. BI.ANGHet, Invenlaire des mosaïques de la Gaule, II (1909), no 1426.

(4) Photographie obligeamment communiquée par la Direction du Musée historique bernois. 


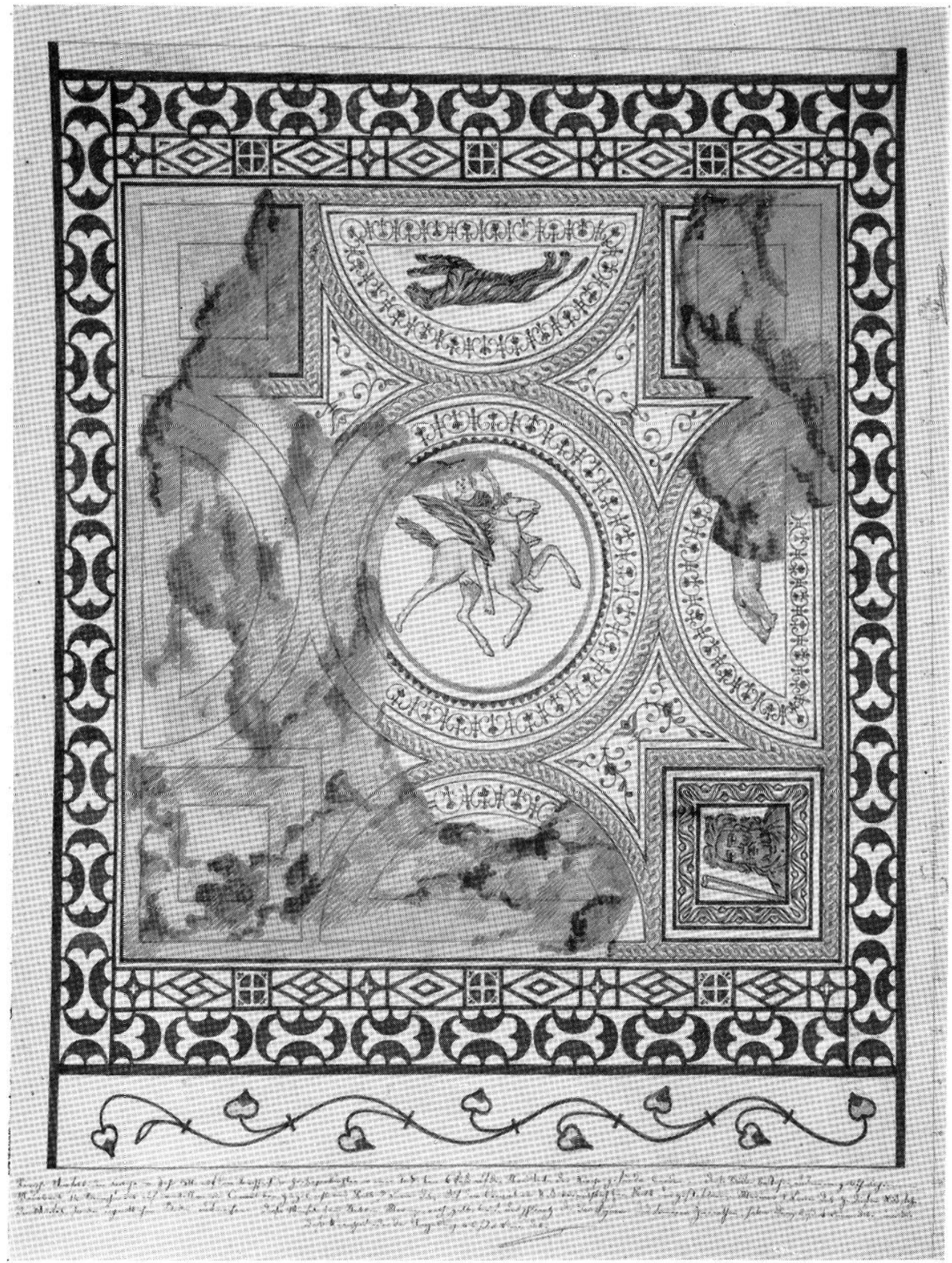

Fig. 2. - Mosaïque d'Herzogenbuchsee (Suisse). Dessin aquarellé de Stürler, 1811 (Musée historique bernois). 
qu'à gauche on voit le bout de l'aile gauche de Pégase. Son regard est dirigé vers le bas mais il ne tient pas de lance et il n'y a pas de Chimère sous les sabots de sa monture.

Le cavalier, dans ces conditions, peut-il être Bellérophon? La mosaïque perdue d'Avenches, connue seulement par un dessin ${ }^{5}$, montrait dans son carré central Bellérophon sur Pégase : les jambes du cheval, galopant vers la droite, sont allongées, le héros pointe vers le bas sa lance sur un espace blanc. Il n'y a aucun doute que le mosaïste ait eu l'intention de peindre ici la mise à mort de la Chimère et nous pouvons deviner que, quand le pavement fut découvert, le monstre, dont la place est réservée audessous des sabots de Pégase, avait été déjà complètement ou partiellement détruit et que, pour cette raison, le dessinateur ne l'a pas reproduit. Revenant au médaillon de Herzogenbuchsee, nous noterons que l'antérieur droit et le postérieur gauche de Pégase, au lieu d'être esquissés, comme il serait normal, respectivement derrière l'antérieur gauche et le postérieur droit, pendent bizarement vers le segment inférieur de la circonférence du médaillon. Peut-être cette portion du pavement était-elle moins bien préservée au moment de la découverte que le dessin ne le suggère et le dessinateur l'a-t-il restituée suivant son imagination, sans reconnaittre les restes de la Chimère. Autrement, si le dessin est bien la représentation correcte de l'original, nous devrons supposer que le mosaïste figurait le moment où le monstre n'est pas encore en vue et nous pourrions interpréter de même, naturel-

(5) A. Blanchet, Inventaire, II (1909), no 1.392 et planche (1922). - S. Reinscir, Réperloire de peintures grecques el romaines (1922), p. $180, n^{\circ} 3$. lement, le pavement d' $\Lambda$ venches. Quant à l'absence de la lance sur le médaillon de Herzogenbuchsee, il nous suffit d'un coup d'œil aux mosaïques de Lullingstone $^{6}$ et de Reims ${ }^{7}$ (fiig. 3), où le bois de la lance ressort à peine sur le fond, pour apprécier la facilité avec laquelle un observateur peu attentif aurait pu négliger complètement l'arme du héros. Les probabilités sont, en fait, entièrement

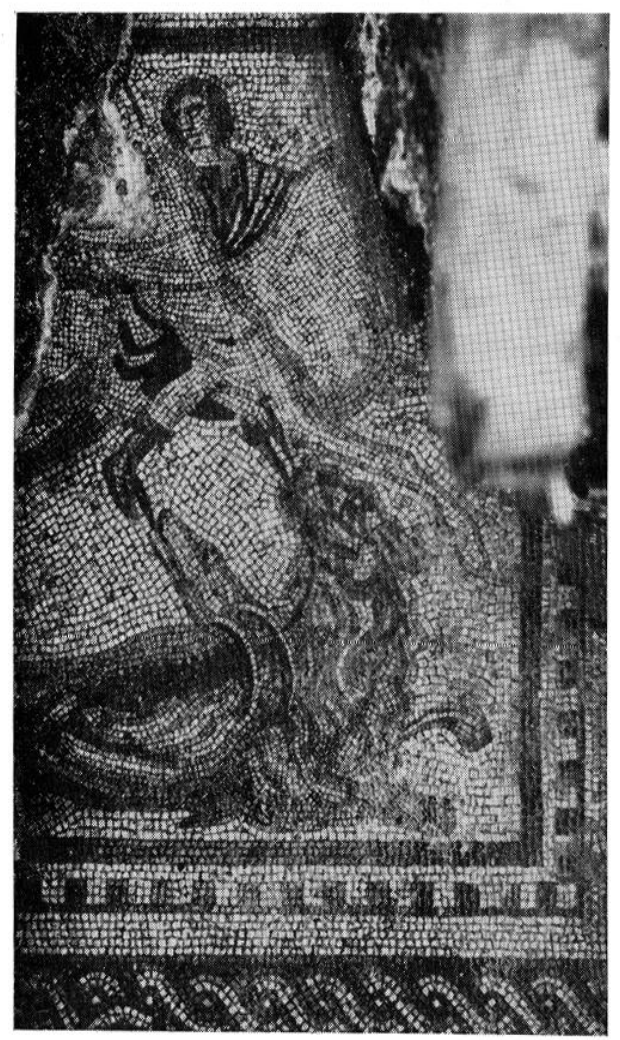

Fig. 3. - Mosaĩque trouvée à Reims en 1938 (Musée de Reims). D'après Sters, Recueil Général des Mosaïques de la Gaule, $\mathrm{n}^{\circ} 6$.

(6) V. supra, n. 2.

(7) H. Stern, Recueil Général des Mosaíques de la Gaule, I, $1^{\text {re }}$ partie, Belgique de l'Ouest (X $\mathrm{X}^{\mathrm{e}}$ supplément à Gallia, 1957), nº 6, pl. 3-4. Je remercie M. Stern de m'avoir aimablement communiqué les photographies de cette mosaïque alors qu'elle était encore inédite. 
favorables à l'insertion du pavement de Herzogenbuchsee dans la série des mosaiques de Bellćrophon, dont il porte à duuze le nombre total des exemplaires connus ${ }^{8}$.

Jocelyn M. G. Toynbee, Museum of Classical Archaeology, Cambridge.
(8) Le pavement sera republié en détail dans l'Inventaire attendu des mosaiques romaines de la Suisse par Mile Victorine von Gonzenbach, qui m: a aimablement donné des renseignements détaillés à son sujet. - Rappelons les provenances des douze pavements connus : Olynthe, Autun, Avenches, Nîmes, Reims, Herzogenbuchsee, Parndorf, Coïmbra, Gerona, Lullingstone, Constantinople et Ravenne.

\section{La Villa gallo-romaine de Guiry-Gadancourt (Seine-et-Oise)}

Les vestiges d'époque romaine signalés dans les publications régionales, sur la base desquelles le regretté Maurice Toussaint a établi son Répertoire Archéologique du Département de Seine-et-Oise', ne donnent qu'une idée insuffisante du degré d'occupation à cette époque de la partie du Vexin comprise entre l'Oise et l'Epte. Sur le territoire de la commune de Guiry (ou des communes limitrophes), où le groupe Archéologique du Camping Club de France a établi le centre de ses activités depuis quelques annćes ${ }^{2}$, les fragments de tegulae et les tcssons de poteries caracléristiques se rencontrent en surface en des points relativement nombreux : dans un rayon d'à peine 2 kilomètres autour de l'agglomération de Guiry nous avons noté une dizaine de points offrant des indices de cette nature correspondant, à coup sûr, à autant d'établissements gallo-romains. L'emplacement choisi pour les fouilles est situé au lieudit "Le Ghemin de Gadancourt ", commune de GadancourtSection Z - parcelle 23, avec extension sur les parcelles 21,22 et 24 , ainsi que

(1) Paris, 1951.

(2) Cf. Galiria, XV, 1957, p. 161, Informalions de M. A. Piganiol, à qui nous adressons l'expression de notre respectueuse gratitude pour le constant appui qu'il veut bien apporter à nos travaux. sur la commune de Guiry-Section Z parcelle 53. Mais, avant le remembrement intervenu en 1952, qui a amené une modification de la limite intercommunale, toute la terre qui compose ces parcelles appartenait à la commune de Guiry et le nom du lieudit figurant au cadastre était celui, beaucoup plus évocateur au point de vue archéologique, de : "Les Terres Noires", qui s'est conservé dans l'usage courant. L'ancienne voie romaine de Paris à Rouen, encore reconnaissable sur le terrain et dite "Chaussée Jules César " passe au NordEst à environ 850 mètres du centre de la zone des fouilles. Des vestiges antiques divers avaient déjà été remarqués dans ce secteur à la fin du $\mathrm{xIx}^{\mathrm{e}}$ siècle$^{3}$, mais n'avaient pas suscité de fouilles systématiques ni même d'observations tant soit peu précises.

Lc site est un vaste plateau dénudé, descendant en pente très douce vers la Chaussée et contrastant avec la vallée verdoyante et boisée où s'est établi le village de Guiry à 1700 mètres au SudOuest. Les fouilles entreprises fin août 1955 ont pris fin, pour ce premier chantier, en novembre 1956, date à laquelle le terrain exploré a été remis en état de

(3) Cf. Manuscrits Léon Plancouard à la mairie de Magny-en-Vexin. 